\title{
Circulating levels of GDF15, MMP7 and miR-200c as a poor prognostic signature in gastric cancer
}

\author{
Moisés Blanco-Calvo ${ }^{\ddagger}$, , Nuria Tarrío ${ }^{\ddagger}, 2$, Margarita Reboredo ${ }^{3}$, Mar Haz-Conde ${ }^{1}$, Jorge \\ García $^{3}$, María Quindós ${ }^{3}$, Angélica Figueroa ${ }^{1}$, Luis Antón-Aparicio ${ }^{1,3}$, Lourdes Calvo ${ }^{3}$ \\ \& Manuel Valladares-Ayerbes, ${ }^{1,3}$ \\ ${ }^{1}$ Translational Cancer Research Department, La Coruña Biomedical Research Institute (INIBIC), Carretera del \\ Pasaje s/n, 15006 La Coruña, Spain \\ ${ }^{2}$ Clinical Biochemistry Department, Marqués de Valdecilla University Hospital, Avenida Valdecilla 25, 39008 \\ Santander, Spain \\ ${ }^{3}$ Clinical Oncology Department, La Coruña University Hospital (CHUAC), As Xubias 84, 15006 La Coruña, Spain \\ ${ }^{*}$ Authors contributed equally
}

\begin{abstract}
:
Aim: To analyze GDF15 and MMP7 serum levels as diagnostic biomarkers in gastric cancer (GC) patients. The prognostic value of GDF15 and MMP7 serum levels in combination with miR-200c blood expression was also analyzed. Patients \& methods: Fifty-two GC and 23 control samples were included. Results: GDF15 and MMP7 proved to be powerful tools for GC diagnosis. Increased levels of GDF15 and MMP7 were associated with shorter progression-free survival and overall survival in univariate analysis. In multivariate analysis, the combination of high levels of GDF15, MMP7 and miR-200c was an independent predictor for death $(\mathrm{p}=0.033)$. Conclusion: GDF15 and MMP7 serum levels have diagnostic value for GC. The combination marker formed by GDF15, MMP7 and miR$200 \mathrm{c}$ is indicative of adverse evolution in GC patients.
\end{abstract}

\section{Keywords:}

Biomarkers; diagnostic; gastric cancer; GDF15; metastasis; miR-200c; MMP7; prognostic 
Gastric cancer (GC) is among the most frequent causes of cancer death worldwide. The stage at diagnosis and the options for curative surgery remain the most important prognostic factors. However, GC shows extensive tumor invasion and early spread, thus, metastasis and locoregional relapses frequently occur despite resection and multimodality therapy. Primary cancer cells are released from their initial sites and spread via the peripheral circulation to the premetastatic niches at distant organs. In this process, both circulating tumor cells (CTC) and host factors can determine whether metastasis and/or recurrence are likely after treatment. The survival of CTC is affected by mediators released by cancer, immune cells and/or stromal cells [1-3] and a variety of circulating factors can create a supportive microenvironment and promote the survival of metastatic seeds [4].

Recently, several studies have demonstrated that the levels of specific members of the miR-200 family of miRNAs are increased in blood of patients with different epithelial cancers compared with healthy controls. Furthermore, increasing levels of circulating miR-200b and miR-200c have been correlated with unfavorable prognosis in breast cancer [5] and GC [6] patients, respectively.

The miR-200 miRNA family has been shown to regulate the epithelial-mesenchymal plasticity that may be crucial at different stages of metastasis through direct targeting of the ZEB-Cadherin 1 axis [7-9]. The knowledge about the modulation of this axis and the epithelial-mesenchymal transition (EMT) was recently expanded with the addition of TGF- $\beta$, an important regulator of inflammation. The autocrine TGF- $\beta$ signaling is necessary for the maintenance of high levels of ZEB proteins and the downregulation of miR-200 miRNA family in cells with mesenchymal phenotype [10]. Additional evidences support the connection between miR-200c and the inflammatory and prometastatic microenvironment in tumors. Thus, a recent study showed the existence of a feed-forward inflammatory signaling system initiated by the miR-200c suppression mediated by the proinflammatory cytokine IL6 [11]. Interestingly, recent evidences support the idea that IL6 could regulate the expression of GDF15 in prostate cancer [12]. Also, the targeting of SEC23A, an essential component of COPII secretory vesicles, by the miR-200 family promotes metastasis by influencing on the whole secretome, including the extracellular matrix components, proinflammatory cytokines and antimetastatic proteins [13]. Finally, it has been recently demonstrated that miRNAs can functioning as ligands for TLR8, thus triggering a prometastatic inflammatory response [14].

Diverse clinical parameters indicating a proinflammatory host response [15] and the serum levels of several soluble factors have shown to correlate with poor prognostic in GC patients. However, there have been few efforts to identify associations between RNA markers in blood, serum levels of inflammatory and proinvasive mediators, and prognosis in GC patients. To address this issue, we analyzed the serum levels of GDF15 and MMP7 in a cohort of GC patients in which the blood miR-200c levels had been previously determined [6]. We choose GDF15 and MMP7 for analysis because these proteins have not comprehensively analyzed in relation to their prognostic role in GC.

GDF15, also known as MIC1 [16], NAG1, PLAB, PTGFB and PDF, is a dimeric cytokine belonging to TGF- $\beta$ superfamily involved in the regulation of macrophage activation [17]. Expression of GDF15 can only be physiologically detected during development in placenta and some others embryonic tissues [17], which increases its interest as tumor marker. To date, GDF15 expression was related to a large number of pathological conditions such as renal [18] and cardiovascular diseases [19], and cancer [20]. In malignant diseases, alterations in the GDF15 expression was correlated with poor prognosis in metastatic melanoma [21] and prostate cancer [22]. In addition, increased levels of GDF15 in different body fluids were associated with worse outcome in glioblastoma [23] and in ovarian [24], endometrial [25], prostatic [26], pancreatic [27] and colorectal cancer [28]. Regarding GC, the overexpression and exposure to exogenous GDF15 has been involved in the activation of different proliferative and invasive signals in tumor cell lines [29,30], whereas the GDF15 expression in primary tumors was correlated with progressive pathological parameters [31]. Finally, GDF15 levels were detected significantly increased in serum and plasma from GC patients versus healthy controls [32,33]. Increased GDF15 plasma levels were associated with poor survival in a pool of esophageal and GC patients including different histological subtypes, although without achieving prognostic independence [33]. 
MMP7 [34] is a secreted matrix metalloproteinase (MMP) belonging to the large family of zinc- and calcium-dependent endopeptidases. MMPs are responsible for the degradation of extracellular matrix components and are involved in important physiological processes such as tissue remodeling [35]. In cancer, the abnormal activity of MMPs [36], including MMP7 [37], has been related to proliferation, angiogenesis and enhanced spreading ability of tumor cells, with the consequent disease progression, metastasis and poor prognosis. The role of MMPs as biomarkers has been extensively studied in different types of cancer [38], including GC [39]. Regarding $M M P 7$, several authors have correlated its expression in gastric primary tumors with several clinicopathological variables [40-42] and survival [43,44]. However, the MMP7 serum levels were only studied as prognostic factor in combination with MMP3 in a cohort of Helicobacter pylori-infected GC patients, but without obtaining significance in multivariate analysis [45].

Therefore, we hypothesized that the serum levels of GDF15 and MMP7, as proinflammatory and prometastatic proteins, might correlate with GC diagnosis, staging and prognosis, and could be useful as clinical biomarkers in GC. In addition, the strength to predict progression-free survival (PFS) and overall survival (OS) of miR- 200c expression in combination with this protein signature in blood was investigated across $52 \mathrm{GC}$ cases. Our results suggest that this blood profile defines a promising prognostic signature in GC.

\section{Patients \& methods}

\section{Patients \& controls}

Consecutive GC patients were recruited at La Coruna University Hospital (CHUAC, Spain). Inclusion and exclusion criteria have been previously described [6]. Briefly, a confirmed pathological diagnosis of gastric or gastroesophageal junction adenocarcinoma and no prior systemic medical therapy for cancer were required.

The diagnostic work-up included a clinical examination, blood sampling, endoscopy (when clinically indicated), and CT scanning of chest, abdomen and pelvis. Tumors and regional lymph nodes collected during surgery were processed on a routine diagnostic basis. When surgery was not performed, pathological diagnostic was based on endoscopic or radiological-guided biopsies. Staging and pathological analyses have been previously described [6]. To monitor disease progression, patients were followed-up clinically and with imaging every 8-12 weeks during the first 2 years and every 6 months thereafter.

The controls were recruited from the patients' family and relatives. We only excluded subjects with a previous history of malignant disease. Thus, controls with different chronic but stable diseases (e.g., peptic disease, hypertension, diabetes mellitus or heart disease) were eligible and consecutively recruited. The control cohort was selected to include a sex and age distribution that was comparable to the patient group.

This study was approved by the institutional research ethics committee and conducted in compliance with the Declaration of Helsinki. Written informed consents were obtained from all the patients and the controls prior to their inclusion in the study. 


\section{Blood sampling \& serum determinations}

Peripheral venous bloods were obtained after surgery or in the presence of clinical and radiological disease when surgery was not indicated and before any systemic chemotherapy treatment. Blood samples were immediately processed by centrifugation $\left(1500 \times \mathrm{g}, 10 \mathrm{~min}, 4^{\circ} \mathrm{C}\right)$ for serum isolation. Serum samples were stored at $-80^{\circ} \mathrm{C}$ until their use. After the first thawing cycle, samples were assessed in duplicate for GDF15 and MMP7 levels using commercially available quantitative sandwich ELISA kits (Quantikine Colorimetric Sandwich ELISA kit, R\&D Systems, MN, USA) following the procedures indicated by the manufacturer. The minimum detectable levels indicated by manufacturer for GDF15 and MMP7 were $2.0 \mathrm{pg} / \mathrm{ml}$ (range: $0.0-4.4 \mathrm{pg} / \mathrm{ml}$ ) and $0.016 \mathrm{ng} / \mathrm{ml}$ (range: $0.005-0.094 \mathrm{ng} / \mathrm{ml}$ ), respectively. Optical densities were quantified by using a microtiter plate reader spectrophotometer (MultiSkanPlus Plate Reader, Labsystem Thermo Scientific, MA, USA). GDF15 and MMP7 analyses were performed with no knowledge of the clinical or follow-up data. The quantitative detection of miR-200c in blood has been previously described [6].

\section{Bioinformatic analysis}

The Search Tool for the Retrieval of Interacting Genes/Proteins (STRING) database [46] was used to detect known and predicted interactions between GDF15 and MMP7 and their role on hypothetical regulatory or signaling networks. Microarray expression data were extracted using the Gene Expression Atlas tool from the European Bioinformatic Institute [47]. TargetScan version 6.2 (Whitehead Institute for Biomedical Research, MA, USA) was used to detect hypothetical miRNA binding sites on the 3'-UTR of analyzed genes [48].

\section{Study design \& statistical analyses}

This study was intended to establish the usefulness of GDF15 and MMP7 serum levels as clinical biomarkers and to determine its potential prognostic value in GC patients. The study was performed following the proposed guidelines of the Early Detection Research Network [49]. The design and results are presented in accordance with the REMARK guidelines [50].

The receiver operating characteristic (ROC) curves were constructed for both markers by plotting sensitivity (y-axis) versus 1-specificity (x-axis) and the areas under the curves (AUCs) were calculated. The diagnostic performance including sensitivity, specificity, positive and negative predictive values, and accuracy of GDF15 and MMP7 serum quantifications were also estimated [49]. The potential correlations between GDF15 and MMP7 serum levels and the clinical and pathological features of the study subjects were analyzed. The normality of the distribution of GDF15 and MMP7 levels was determined using the Kolmogorov-Smirnov test. Thus, parametric or nonparametric statistics were used, as appropriate. The relationships between GDF15, MMP7 and miR-200c levels were analyzed.

PFS was measured as the time between the baseline blood sampling and the documentation of first tumor progression, based on clinical and radiological findings, or death (events). OS was measured from the time at which the baseline blood sample was obtained to the date of death from any cause or date of last follow-up.

The patients who were alive and progressionfree at the time of analysis were censored by using the time between the blood assessment and their most recent follow-up evaluations. The distributions of timeto-event end points, namely PFS and OS, were estimated using the Kaplan-Meier method and compared using the log-rank test. 
The optimal prognostic cutoff values for GDF15 and MMP7 were determined by using the X-tile software (Yale School of Medicine, CT, USA) [51]. The X-tile software provides a method of dividing a single cohort into training and validation subsets, when separate training and validation cohorts are not available. In addition, standard Monte Carlo crossvalidation is performed to produce corrected p-values to assess statistical significance of data assessed by multiple cut-points.

Multivariate survival analyses (PFS and OS) were performed using Cox regression models. We estimated hazard ratios (HRs), $95 \%$ CIs and p-values.

The statistical power of the study was estimated post-hoc, taking into account a Kaplan-Meier estimated median OS of 40 weeks and 87 weeks, in the 'poor-prognostic signature' group and in the rest of the cohort, respectively, and an $\alpha$-error of 0.05 . The poor-prognostic signature was defined by increased blood expression of GDF15, MMP7 and miR-200c. The observed probability of survival at 2 years was 0.75 in the low-risk signature group and 0.25 in the high-risk signature group. With the sample size of 52 patients, the study was able to demonstrate by two-sided log-rank test, a significant difference in OS, with a statistical power higher than $90 \%$.

All statistical tests were two-sided and p-values less than 0.05 were considered significant. SPSS Statistics (IBM Corporation, NY, USA) and GraphPad Prism (GraphPad Software, CA, USA) were used for data analyses.

\section{Results}

Patients \& clinical data

From November 2006 to July 2010, 52 GC patients and 23 healthy controls were consecutively enrolled. The clinical and pathological characteristics of patients are shown in Table 1. The composition of the population of patients included in this study reflects the disease state in which patients come to the medical offices in our department. Considering the lack of screening programs for GC in the country where participants have been recruited, the diagnosis is usually performed in patients with locally advanced or metastatic disease (stages III or IV). The mean age of controls was 61.87 year (standard error of mean [SEM]: 1.568) while the mean age of patients was 65.94 years (SEM: 1.315; $\mathrm{p}=0.073$, Student's $t$-test). The ratio of males to females was similar among controls and patients ( $\mathrm{p}=0.068$; Pearson's $\chi^{2}$ test).

We performed follow-up of all patients enrolled until their death or the end of study (mean: 76.52 weeks; SEM: 8.3). The last date of follow-up for the survivors was 5 September 2010. The mean followup time of survivors was 118.9 weeks (SEM: 14.63 weeks; median: 104; range: 28-232). Tumor progression was detected in 38 patients $(73.1 \%)$ and $35(67.3 \%)$ died of advanced disease. Seven relapse events occurred among stage I-III patients while 31 patients experienced progression of metastatic disease.

The median PFS was 27.5 weeks (range: 0-232) and the median OS was 62 weeks (range: 2-232). 
Table 1. Patients and clinicopathological data.

\begin{tabular}{|c|c|c|}
\hline & $\mathrm{n}$ & $\%$ \\
\hline \multicolumn{3}{|l|}{ Age (years) } \\
\hline Mean (SEM) & $65.94(1.31)$ & \\
\hline Range & $42-85$ & \\
\hline Quartile $1(<59)$ & 15 & 28.85 \\
\hline Quartile 2 (59-66) & 12 & 23.08 \\
\hline Quartile $3(66-74.75)$ & 12 & 23.08 \\
\hline Quartile $4(>74.75)$ & 13 & 25.00 \\
\hline \multicolumn{3}{|l|}{ Gender } \\
\hline Male & 42 & 80.8 \\
\hline Female & 10 & 19.2 \\
\hline \multicolumn{3}{|l|}{$E C O G$} \\
\hline $0-1$ & 37 & 71.2 \\
\hline 2 & 10 & 19.2 \\
\hline \multicolumn{3}{|l|}{ Tumor location } \\
\hline Proximal & 13 & 25.0 \\
\hline Distal & 36 & 69.2 \\
\hline Multicentric & 3 & 5.8 \\
\hline \multicolumn{3}{|l|}{ Stage } \\
\hline $\mathrm{I}-\mathrm{II}$ & 9 & 17.3 \\
\hline III & 12 & 23.1 \\
\hline IV & 31 & 59.6 \\
\hline \multicolumn{3}{|l|}{$p T$} \\
\hline pT1-2 & 8 & 15.4 \\
\hline pT3 & 19 & 36.5 \\
\hline pT4 & 7 & 13.5 \\
\hline pTx & 18 & 34.6 \\
\hline \multicolumn{3}{|l|}{$p N$} \\
\hline pNO & 9 & 17.3 \\
\hline pN1 & 11 & 21.2 \\
\hline $\mathrm{pN} 2$ & 9 & 17.3 \\
\hline $\mathrm{pN} 3$ & 4 & 7.7 \\
\hline \multicolumn{3}{|l|}{ Histology } \\
\hline Intestinal & 28 & 53.8 \\
\hline Diffuse & 21 & 40.4 \\
\hline Mixed & 3 & 5.8 \\
\hline \multicolumn{3}{|l|}{$R$} \\
\hline R0 & 20 & 38.5 \\
\hline $\mathrm{R} 1-2$ & 32 & 61.5 \\
\hline \multicolumn{3}{|l|}{$M$} \\
\hline None $(0)$ & 23 & 44.2 \\
\hline Liver (1) & 8 & 15.4 \\
\hline Non liver (1) & 13 & 25.0 \\
\hline Both (1) & 8 & 15.4 \\
\hline \multicolumn{3}{|l|}{ Grade } \\
\hline Low $(0-1)$ & 21 & 40.4 \\
\hline High (3-4) & 27 & 51.9 \\
\hline \multicolumn{3}{|c|}{ Vascular/perineural invasion } \\
\hline No & 11 & 21.2 \\
\hline Yes & 19 & 36.5 \\
\hline Unknow & 22 & 42.3 \\
\hline
\end{tabular}

pT, pN, M and staging as indicated in [66].

ECOG: Eastern Cooperative Oncology Group performance status (as indicated in [52]); R: Residual disease, as indicated in text; SEM: Standard error of mean. 
The determination of serum levels of GDF15 and MMP7 (Figure 1) was performed using 75 samples (52 patients and 23 controls). The medians for GDF15 serum levels were $453.36 \mathrm{pg} / \mathrm{ml}$ (range: 147.651576.18) and $212.22 \mathrm{pg} / \mathrm{ml}$ (range: 90.28-429.44) for cancer patients and controls, respectively (p < 0.001; Mann-Whitney U test). The medians for MMP7 serum levels were $3.27 \mathrm{ng} / \mathrm{ml}$ (range: $1.04-11.31$ ) in cancer patients and $1.73 \mathrm{ng} / \mathrm{ml}$ (range: 0.66-3.43) in controls ( $\mathrm{p}<0.001$ ). The median for GDF15 serum levels was $359.12 \mathrm{pg} / \mathrm{ml}$ (range: 186.47-1556.47) in stage I-III patients while in stage IV patients was $703.24 \mathrm{pg} / \mathrm{ml}$ (range: $147.65-1576.18 ; \mathrm{p}=0.004$ ). For MMP7, the medians of serum levels were 2.52 $\mathrm{ng} / \mathrm{ml}$ (range: $1.04-5.37 \mathrm{ng} / \mathrm{ml}$ ) in stage I-III patients and $4.78 \mathrm{ng} / \mathrm{ml}$ (range: $1.39-11.31$ ) in stage IV patients $(\mathrm{p}=0.002)$.



Figure 1. GDF15 and MMP7 serum levels. (A) GDF15 and (B) MMP7 serum levels in healthy controls, stage I-III and stage IV gastric cancer patients. Lines in each group represent the respective median values. p-values correspond to Mann-Whitney U tests.

Comparing the serum levels in patients and controls, the AUCs were 0.8796 (95\% CI: 0.804-0.955; $\mathrm{p}<0.0001$ ) and 0.8597 (95\% CI: 0.774-0.942; $\mathrm{p}<0.0001$ ), respectively, for GDF15 and MMP7 (Figure 2). Based on ROC curve analysis, the optimal discrimination between patients and healthy individuals was achieved using $325.28 \mathrm{pg} / \mathrm{ml}$ as a cutoff value for GDF15 serum levels. Using this cut-point, we obtained a sensitivity of $73.08 \%$ and a specificity of $91.30 \%$. In the case of MMP7, the optimal cutoff value to distinguish between patients and healthy individuals was set at $2.13 \mathrm{ng} / \mathrm{ml}$, with a sensitivity of $78.85 \%$ and a specificity of $86.96 \%$ (Table 2). 
Table 2. Diagnostic performance GDF15 and MMP7 serum levels.

\begin{tabular}{|c|c|c|c|c|c|}
\hline & Ss $(95 \%$ CI ) & $\mathrm{Sp}(95 \% \mathrm{CI})$ & PPV (95\% CI ) & NPV (95\% CI ) & Accuracy $(95 \%$ CI ) \\
\hline \multicolumn{6}{|c|}{ Patients vs controls } \\
\hline GDF15 > 325.28 & $73.08(58.73-84.00)$ & $91.30(70.49-98.48)$ & $95.00(81.79-99.13)$ & $60.00(42.21-75.65)$ & $78.67(67.39-86.96)$ \\
\hline MMP7 > 2.13 & $78.85(64.91-88.48)$ & $86.96(65.33-96.57)$ & $93.18(80.29-98.22)$ & $64.52(45.38-80.17)$ & $81.33(70.33-89.06)$ \\
\hline \multicolumn{6}{|l|}{ Stage I-III vs IV } \\
\hline GDF15 >532.05 & $67.74(48.54-82.68)$ & $90.48(68.17-98.33)$ & $91.30(70.49-98.48)$ & $65.52(45.66-81.40)$ & $76.92(62.83-87.02)$ \\
\hline MMP7 >3.79 & $61.29(42.29-77.58)$ & $90.48(68.17-98.33)$ & $90.48(68.17-98.33)$ & $61.29(42.29-77.58)$ & $73.08(58.73-84.00)$ \\
\hline \multicolumn{6}{|c|}{ Controls vs stage I-II } \\
\hline GDF15 > 294.40 & 88.89 (50.67-99.42) & $82.61(60.45-94.28)$ & $66.67(35.44-88.73)$ & $95.00(73.06-99.74)$ & $\begin{array}{l}84.38(66.45- \\
94.10)\end{array}$ \\
\hline MMP7 > 2.30 & $66.67(30.92-90.96)$ & $91.30(70.49-98.48)$ & $75.00(35.58-95.55)$ & $87.50(66.54-96.71)$ & $84.38(66.45-94.10)$ \\
\hline
\end{tabular}

GDF15 serum levels are presented in pg/ml and MMP7 serum levels are in ng/ml.

NPV: Negative predictive value (in \%); PPV: Positive predictive value (in \%); Sp: Specificity; Ss: Sensitivity.

ROC curves were also used to assess the ability of GDF15 and MMP7 serum levels to discriminate between stage I-III and stage IV patients. The AUCs obtained were 0.740 (95\% CI: $0.599-0.882 ; \mathrm{p}=$ 0.003 ) and $0.753(95 \% \mathrm{CI}: 0.622-0.885 ; \mathrm{p}=0.002)$ for GDF15 and MMP7, respectively (Supplementary Figure 1; see online at www.futuremedicine.com/doi/suppl/10.2217/ fon.13.263). The optimal GDF15 cutoff value that distinguishes between stage I-III and IV patients was $532.05 \mathrm{pg} / \mathrm{ml}$ with $67.74 \%$ sensitivity and $90.48 \%$ specificity. Using a cutoff value of $3.79 \mathrm{ng} / \mathrm{ml}$ for MMP7 serum levels, the sensitivity and specificity were 61.29 and $90.48 \%$ respectively (Table 2 ).

In addition, GDF15 and MMP7 were also capable to detect patients at early stages of GC. The AUCs for ROC curves distinguishing healthy controls from stage I-II patients were 0.884 (95\% CI: $0.755-$ 1.013; $\mathrm{p}<0.001$ ) for GDF15 and 0.768 (95\% CI: 0.561-0.975; p = 0.020) for MMP7 (Supplementary Figure 1). The optimal cutoff value for GDF15 $(294.40 \mathrm{pg} / \mathrm{ml})$ allowed the detection of early-stage GC patients with $88.89 \%$ sensitivity and $82.61 \%$ specificity (Table 2). MMP7 reached $66.67 \%$ sensitivity and $91.30 \%$ specificity detecting early stage GC patients when the optimal cutoff value was set at $2.30 \mathrm{ng} / \mathrm{ml}$ (Table 2).

Clinical \& pathological characteristics of GDF15 \& MMP7 serum levels

The GDF15 and MMP7 serum levels in relation to clinical and pathological characteristics of patients are shown in Table 3. An increased serum level of GDF15 and MMP7 were associated with Eastern Cooperative Oncology Group (ECOG) performance [52] status 2 ( $p<0.001$ and $p=0.006$, respectively), residual ( $R 1$ and $R 2)$ disease $(p<0.001$ and $p=0.002$, respectively), and advanced (IV) stage $(\mathrm{p}=0.004$ and $\mathrm{p}=0.002$, respectively). The patients with hepatic metastasis showed higher GDF15 and MMP7 serum levels than patients without metastasis or with extrahepatic metastasis only $(\mathrm{p}<0.001)$. 
Table 3. GDF15 and MMP7 serum levels and clinicopathological parameters

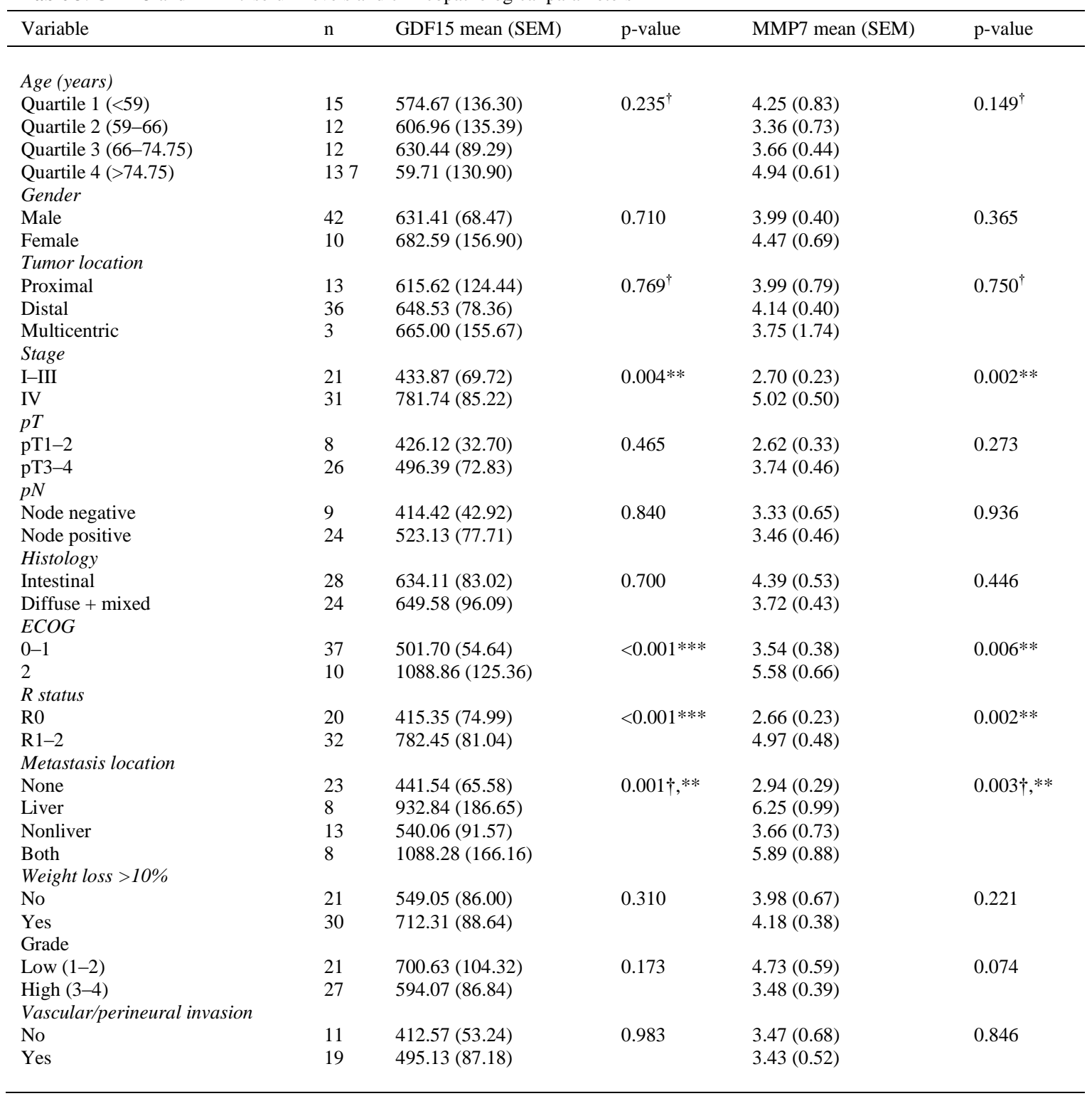

GDF15 serum levels are presented in pg/ml and MMP7 serum levels are in ng/ml. pT, pN and staging as indicated in [66]

$\dagger$ Kruskal-Wallis tests for GDF15 (pg/ml) and MMP7 (ng/ml) serum levels.

$* * 0.001 \leq \mathrm{p}<0.01 ; * * * \mathrm{p}<0.001$.

ECOG: Eastern Cooperative Oncology Group performance status; R: Residual disease (as indicated in text); SEM: Standard error of mean.

There was no correlation between GDF15 and MMP7 either with serum LDH levels, total proteins or weight loss. However, GDF15 and MMP7 were found correlated with ALP (respective Spearman's $\rho$ coefficients and p-values: $0.290, p=0.037$ for GDF15; and 0.402, $p=0.003$ for MMP7) and albumin (respective Spearman's $\rho$ coefficients and p-values: $-0.560, \mathrm{p}<0.001$ for GDF15; and $-0.352, \mathrm{p}=0.011$ for MMP7). GDF15 and MMP7 serum levels were highly correlated (Spearman's $\rho$ coefficient $0.712, \mathrm{p}<$ 0.001) (Table 4). However, the STRING database shows no evidences for direct molecular interactions between both proteins (Supplementary Figure 2). 
Table 4. Correlation of several prognostic factors in gastric cancer and GDF15 and MMP7 serum levels.

\begin{tabular}{lllll}
\hline Variable & \multicolumn{2}{c}{ GDF15 } & & \multicolumn{2}{c}{ MMP7 } \\
\cline { 2 - 3 } \cline { 5 - 6 } & Spearman's $\rho$ & p-value & & Spearman's $\rho$ \\
Weight loss (\%) & 0.160 & 0.263 & 0.219 & 0.122 \\
Positive lymph nodes (n) & -0.160 & 0.398 & -0.090 & 0.636 \\
LDH (U/l) & 0.177 & 0.209 & 0.245 & 0.080 \\
ALP (U/l) & 0.290 & $0.037 *$ & 0.402 & $0.003 * *$ \\
Albumin (g/dl) & -0.560 & $<0.001 * * *$ & -0.352 & $0.011^{*}$ \\
Total proteins (g/dl) & -0.208 & 0.143 & 0.072 & $<18$ \\
GDF15 & - & - & 0.712 & $0.001^{* * *}$ \\
miR-200c & 0.226 & 0.107 & 0.100 & 0.481 \\
\end{tabular}

GDF15 serum levels are presented in pg/ml and MMP7 serum levels are in ng/m 1. miR-200c was measured as relative values of expression as indicated in [6].

$* 0.01 \leq \mathrm{p} \leq 0.05 ; * * 0.001 \leq \mathrm{p}<0.01 ; * * * \mathrm{p}<0.001$

To explore the possible influence of recent surgical procedures on the GDF15 and MMP7 levels, we analyzed their serum concentrations according to the time interval from surgery and blood sampling. The median time from surgery to serum sampling was 6 weeks (mean: 19.1 weeks; SEM: 5.5; range: 2-155). There were no significant differences in GDF15 $(p=0.94)$ and MMP7 $(p=0.22)$ levels according to time intervals ( $<6$ or $\geq 6$ weeks) from the last surgery.

\section{Prognostic significance of GDF15 \& MMP7}

Using the cutoff values provided by X-tile for GDF15 and MMP7 serum levels, patients were divided in two subpopulations with significant differences in PFS and OS. High values of GDF15 (>493.82 $\mathrm{pg} / \mathrm{ml}$ ) were associated with shorter PFS (Figure 3A) and OS (p < 0.001) (Figure 3B). The median PFS in the group with high GDF15 serum levels was 17 weeks (95\% CI: 6-27 weeks) while in the group with low GDF15 serum levels was 53 weeks (95\% CI: 28-102 weeks). The median OS in the group with high GDF15 serum levels were 40 weeks (95\% CI: 22-64 weeks). By contrast, in the group with low GDF15 serum levels, the median OS was 102 weeks (95\% CI: 53-127 weeks).

In the case of MMP7, there were found two different cutoff values providing the greatest differences in PFS $(5.37 \mathrm{ng} / \mathrm{ml})$ and OS $(3.46 \mathrm{ng} / \mathrm{ml})$. MMP7 serum levels higher than indicated were associated with shorter PFS $(\mathrm{p}<0.001)$ (Figure 3C) and OS $(\mathrm{p}=0.003)$ (Figure 3D). The median PFS in the group with high MMP7 serum levels was 10 weeks (95\% CI: 2-27 weeks) while in the group with low MMP7 serum levels was 48 weeks (95\% CI: 23-70 weeks). The median OS in the group with high MMP7 serum levels was 40 weeks (95\% CI: 26-66 weeks). In the group with low MMP7 serum levels, the median OS was 83 weeks (95\% CI: 53-118 weeks).

Then, we performed Cox regression analysis to determine the influence of different clinical and pathological variables, including GDF15 and MMP7 serum values, on the risk of relapse or death. In univariate analysis (Table 5), patients with high serum levels of GDF15 and MMP7, defined by X-tilegenerated cutoff values, had an increased risk of progression (HR [95\% CI]: 3.608 [1.831-7.107], p < 0.001; and 4.172 [2.017-8.629], p<0.001, respectively) and death (HR [95\% CI]: 3.843 [1.799-8.209], p $=0.001 ;$ and $2.602[1.318-5.136], \mathrm{p}=0.006$, respectively). The presence of residual disease and advanced stage were also significantly correlated with progression and death. However, in multivariate analysis (Supplementary Table 1), GDF15 and MMP7, individually or in combination, failed to reach prognostic independence. 




(B)

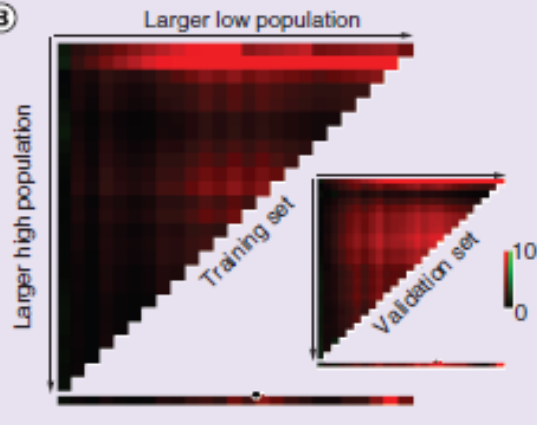

(C)

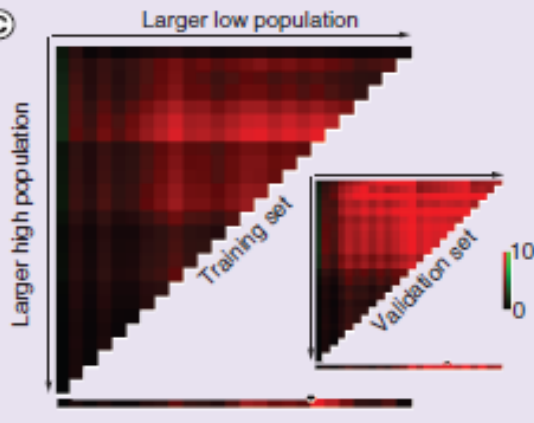

(D)

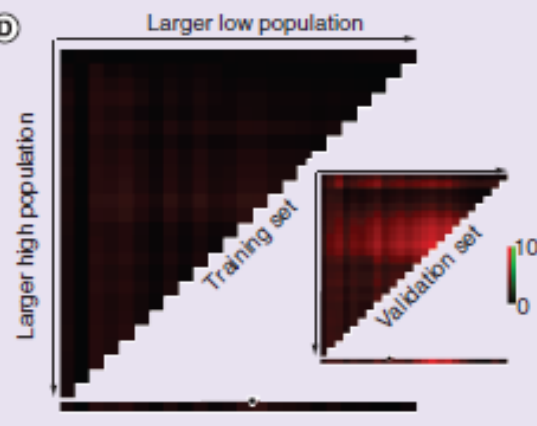


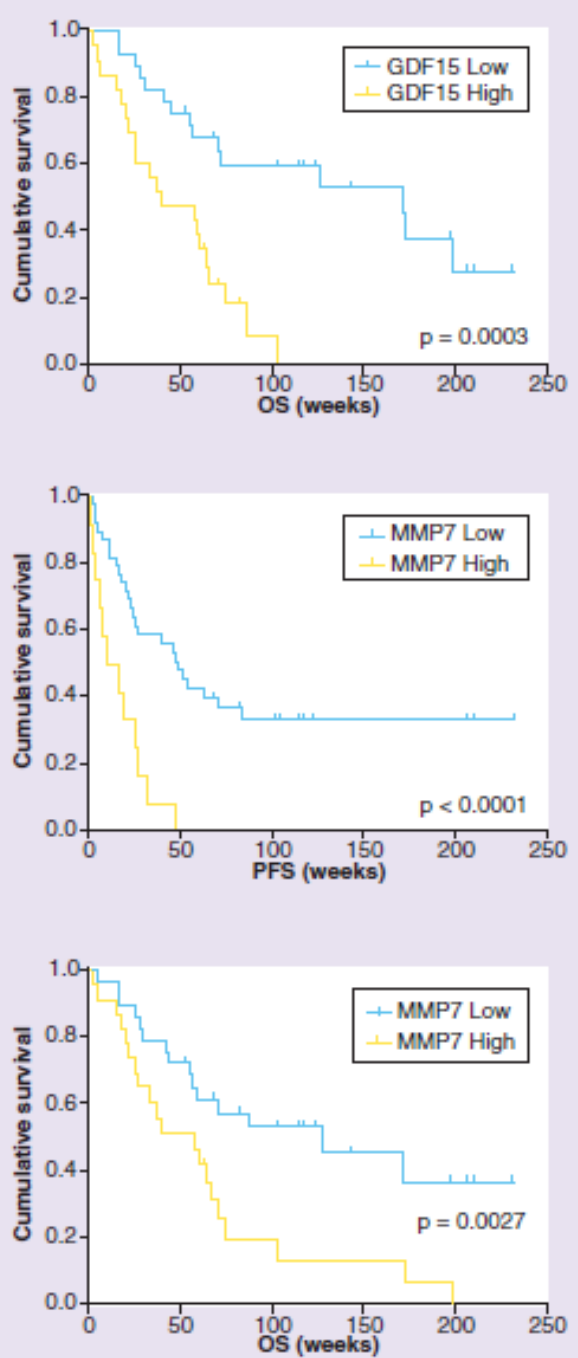

Figure 3. X-tile and survival analysis. X-tile analysis on the training (large heat map) and validation (small heat map) data sets and the respective Kaplan-Meier curves corresponding to subpopulations generated from the entire data set using the different cutoffs provided by X-tile for each biomarker. (A) X-tile and the Kaplan-Meier analysis for GDF15 and PFS, and (B) for GDF15 and OS. (C) X-tile and the Kaplan-Meier analysis for MMP7 and PFS, and (D) for MMP7 and OS. Subpopulations with low values of biomarkers are represented in blue while subpopulations with high values are represented in yellow. Differences between survival curves were assessed by the log-rank test and the corresponding p-values are provided. OS: Overall survival; PFS: Progression-free survival. 
Table 5. Univariate Cox regression.

\begin{tabular}{|c|c|c|c|c|c|}
\hline Variable & Subset & \multicolumn{2}{|c|}{ Progression } & \multicolumn{2}{|l|}{ Death } \\
\hline MMP7 for progression & $\leq 5.37 />5.37$ & $4.172(2.017-8.629)$ & $<0.001 * * *$ & - & - \\
\hline MMP7 for death & $\leq 3.46 />3.46$ & - & - & $2.602(1.318-5.136)$ & $0.006^{* *}$ \\
\hline Residual disease & $\mathrm{R} 0 / \mathrm{R} 1-2$ & $10.953(4.432-27.067)$ & $<0.001 * * *$ & $6.079(2.489-14.847)$ & $<0.001 * * *$ \\
\hline
\end{tabular}

Univariate Cox regression analysis was performed to identify variables influencing on progression and death risk. GDF15 serum levels are presented in $\mathrm{pg} / \mathrm{ml}$ and MMP7 serum levels are in $\mathrm{ng} / \mathrm{ml}$.

$* * 0.001 \leq \mathrm{p}<0.01 ; * * * \mathrm{p}<0.001$.

HR: Hazard ratio.

\section{Combined analysis of miR-200c blood expression \& GDF15 \& MMP7 serum levels}

In a previous work [6], we established the expression of the miRNA miR-200c as an independent prognostic variable in GC, using an aliquot of the same blood samples that we analyze now for GDF15 and MMP7. In that study, the expression levels of miR-200c in blood were associated with shorter PFS (HR [95\% CI]: 2.27 [1.093-4.712]; $\mathrm{p}=0.028)$ and OS (HR [95\% CI]: 2.24 [1.091-4.614]; $\mathrm{p}=0.028$ ) with independence of additional prognostic variables.

Here, we asked for the prognostic capacity of the molecular signature composed by the combination of GDF15 and MMP7 serum levels, and miR-200c blood expression. The increased presence in blood of GDF15, MMP7 and miR-200c defined a 'poor prognostic' signature that was significantly associated with a shorter PFS (log-rank: $p<0.001)$ and a poor OS (log-rank: $p<0.001)$. In the group of GC patients with high levels for all components of this signature, the median PFS and OS were respectively 6 weeks (95\% CI: 0-13.2) and 26 weeks (95\% CI: 18.4-33.6). In the group with low GDF15, MMP7 and miR200c blood levels, the median PFS was 40 weeks (95\% CI: 17.4-62.6) and the median OS was 87 weeks (95\% CI: 42.6-131.4). In order to analyze the potential correlation of this prognostic signature with tumor burden, we have used the LDH and ALP levels as surrogate of tumor load. We did not obtain any significant result when high levels of 'GDF15+MMP7+miR-200c' were assessed in relation to LDH and ALP levels by binary logistic regression. The combination of elevated GDF15 and MMP7 serum levels with high miR- 200c expression in blood was able to predict OS (HR [95\% CI]: 2.521 [1.079-5.891]; $\mathrm{p}=$ 0.033 ) with independence of advanced stage and weight loss $>10 \%$ (Table 6). In line with this finding, the analysis of stage IV patients shows that the presence of the 'poor prognostic' signature is significantly associated with low OS (log-rank: $\mathrm{p}=0.004)$. 
Table 6. Multivariate Cox regression with GDF15 and MMP7 in combination with miR-200c.

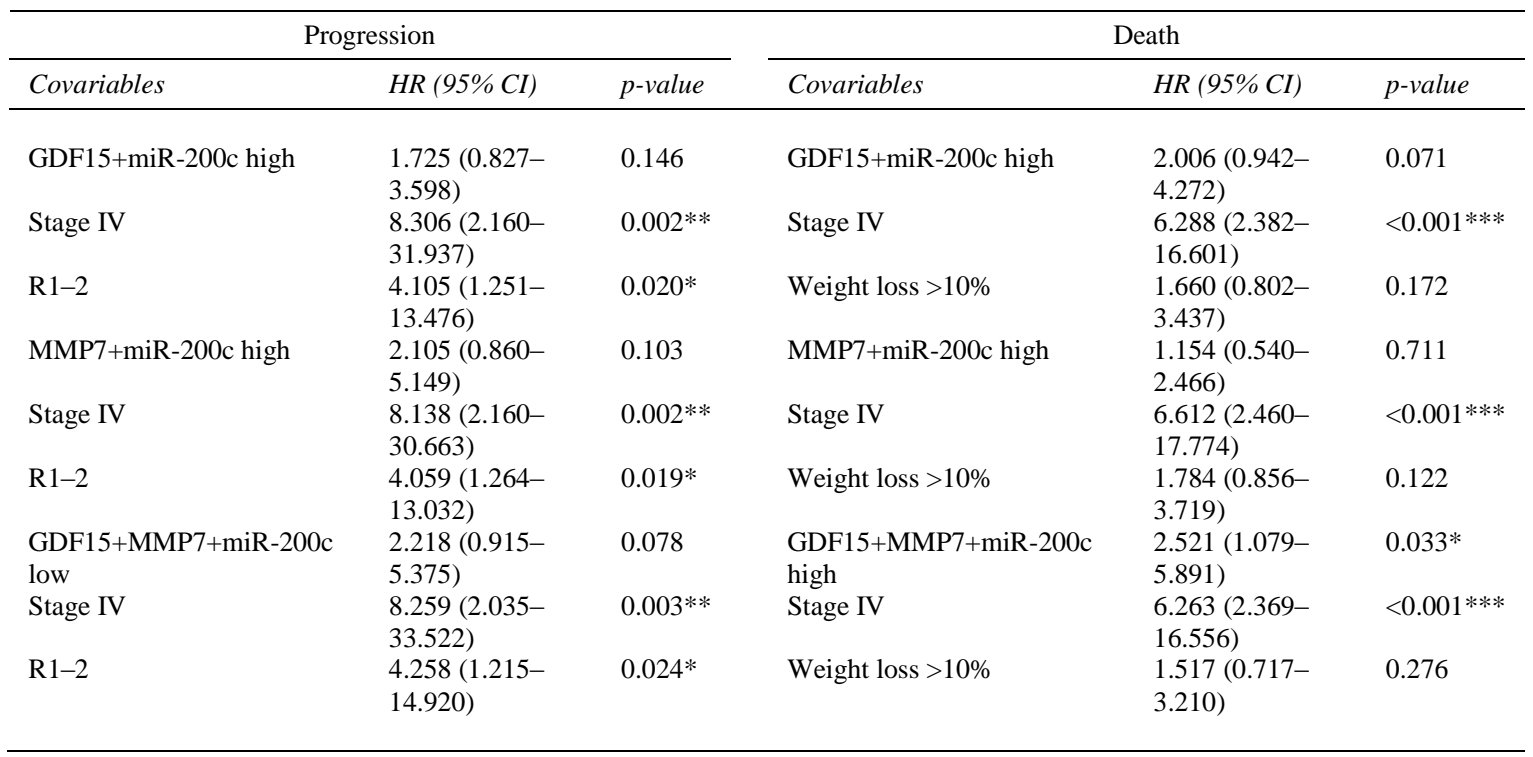

Multivariate Cox regression analysis was performed to identify independent variables influencing on progression and death risk. The analysis was performed for GDF15 and/or MMP7 serum levels in combination with miR-200c blood expression. The covariates included in the model were residual disease (R1-2 vs R0) and stage (early I-III stages vs advanced IV stage) for progression, and weight loss $>10 \%$ (no vs yes) and stage (early I-III stages vs advanced IV stage) for death. miR-200c expression values were categorized using as cutoff the mean expression as previously depicted [6]. GDF15 and MMP7 values were categorized as indicated in text. 'GDF15+miR-200c high' and 'MMP7+miR-200c high' are categorical variables representing values above the respective cutoffs values for all biomarkers. In the variable 'GDF15+MMP7+miR-200c low', used in progression analysis, we grouped values below the respective cutoffs for GDF15, MMP7 and miR-200c. In the variable 'GDF15+MMP7+miR-200c high', used in survival analysis, values above the corresponding cutoffs for all biomarkers were grouped.

$* 0.01 \leq \mathrm{p} \leq 0.05 ; * * 0.001 \leq \mathrm{p}<0.01 ; * * * \mathrm{p}<0.001$

HR: Hazard ratio.

\section{Discussion}

This study analyzes for first time the relationship between the serum levels of two inflammatory and proinvasive proteins, GDF15 and MMP7, and the blood expression level of miR-200c in a cohort of GC patients, with the objective of obtaining a diagnostic and prognostic signature based on microenvironmental and CTC molecular properties. In our first analysis, we found significantly elevated the serum levels of GDF15 and MMP7 in GC patients, as previously demonstrated [32]. Likewise, we found higher serum levels of GDF15 and MMP7 in stage IV GC patients (Figure 1), in line with the findings already performed on colorectal, breast and prostate carcinomas [53]. Taking into account these data and the lack of reliable markers for GC detection [54], we decide to explore the diagnostic performance of GDF15 and MMP7 serum levels as biomarkers for GC (Figure 2), as well as their ability to distinguish early stages from healthy individuals, and advanced stages among GC patients (Supplementary Figure 1). The analysis of the respective ROC curves shows that both GDF15 and MMP7 are reliable candidate biomarkers for diagnostic purposes. However, when used to detect advanced disease, both biomarkers suffered an important decrease in sensitivity (Table 2). This reduction in sensitivity may be attributable to the fact that the serum amount of GDF15 and MMP7 is not different between GC patients with extrahepatic metastasis and nonmetastatic patients. Indeed, when we construct a specific ROC curve for hepatic disease (data not shown), the same cutoff values used for the discrimination of advanced stages now render a sensitivity and specificity of $87.5 \%$ and $75 \%$ for GDF15, and $81.25 \%$ and $77.78 \%$ for MMP7, respectively. These findings suggest that GDF15 and MMP7 might be secreted by liver in response to the colonization by metastatic GC cells. 
Since GDF15 and MMP7 serum levels were not thoroughly examined for prognostic purposes in GC patients in previous studies, we asked for their association with PFS and OS and for their ability to predict risk of relapse or death. The Kaplan-Meier curves using X-tilebased cutoff values for stratification of GDF15 and MMP7 serum levels show that GC patients with high levels of any of both biomarkers have a worse PFS and OS (Figure 3). However, although in univariate analysis both GDF15 and MMP7 serum levels were significantly associated with PFS and OS (Table 5), in multivariate analysis neither GDF15 nor MMP7 were able to independently predict any of these outcomes (Supplementary Table 1). In addition, the Kaplan-Meier analysis shows that high serum levels of GDF15 and/or MMP7 (both or one of biomarkers high) have a similar impact on PFS in GC patients (Supplementary Figure 3). Also, the combined Kaplan-Meier analysis for GDF15 and MMP7 serum levels shows a similar effect on OS when one or both biomarkers have serum levels below the cutoff values (Supplementary Figure 4). The grouped behavior of GDF15 and/or MMP7 positivity in Kaplan-Meier analysis for PFS may be a direct consequence of the high correlation found between both biomarkers (Table 4) and could indicate a physiological association between both biomarkers. However, we obtain an inverse behavior in the OS analysis in which survival in patients with low levels for both biomarkers is similar to survival in patients with one of biomarkers high. This result points, therefore, to a negative synergic effect on survival only when both biomarkers show elevated serum levels. In order to explore the possible relationships between these two proteins, we performed a query to the STRING database [55]. While no evidence for direct interactions was found (Supplementary Figure 2), the text mining tool returns a single publication [56] in which changes in the expression of GDF15 and MMP7 were related to PNN forced overexpression in HEK293 cells. The search in Gene Expression Atlas [47] for microarray data about PNN, GDF15 and $M M P 7$ expression in GC versus adjacent nontumor samples returns no conclusive results relative to their hypothetical joint regulation. Beyond these data, the expression and the potential relation of PNN, GDF15 and $M M P 7$ was not analyzed in detail in GC, so we can only suggest a potential effect of PNN on the overexpression and/or elevated secretion of GDF15 and MMP7. In addition, the expression and secretion of GDF15 and MMP7 in cancer could be affected by the deregulation of other multiple interacting and/or regulatory proteins (Supplementary Figure 2), which hinders the interpretation of any possible relationship. We also wondered for the hypothetical regulation of both proteins by a common miRNA. However, when searching for predicted miRNA binding sites on the $3^{\prime}$-UTR of both genes, using TargetScan 6.2 [48], none of returned results were coincident (data not shown).

Given the proposed effects of GDF15 on adipose tissue, body mass and food intake [57-59], we also explore the association of weight loss and GDF15 serum levels in our cohort of GC patients. However, while GDF15 plasma levels were previously found significantly elevated in esophago-GC patients with cachexia (defined as weight loss $>10 \%$ ) [33], in this study we could not found such difference for GDF15 serum levels in GC patients. Moreover, conversely to previous studies [33], we could not find association of weight loss $>10 \%$ with PFS or OS in univariate analysis (Table 5) and with OS in multivariate analysis (Table 6 \& Supplementary Table 1). In addition, GDF15 serum levels were not correlated with the percentage of weight loss (Table 4). However, such as was before depicted in plasma of esophago-GC patients [33], we found GDF15 serum levels negatively associated with albumin, a marker for nutritional status. This apparent contradiction indicates that weight and nutritional status in GC patients are multifactorial events and that may be influenced by opposite variables.

Nowadays, there are increasing evidences supporting the role that tumor microenvironment plays on the capacity of malignant cells to grow, invade adjacent tissues and spread [60]. The signals arising from the inflammatory component of this microenvironment are among the most influencing factors on the metastatic potential of primary tumors [61,62]. However, the establishment of metastasis lies also on factors associated to target tissues and organs [60,63], beyond those inherent to primary tumors and their capacity of CTC release. Therefore, that a given tumor can spread or not to distant locations in different patients depends on individual molecular features that predispose or protect from metastasis. The secretion of inflammatory, proangiogenic and proinvasive proteins in the metastasis sites is one of events occurring during their colonization by cancer cells [63]. This gives rise to the generation, and consequent release, of different proteins to blood, among which are the MMPs [64] and perhaps GDF15. In fact, it was recently demonstrated that the overexpression of GDF15 is able to restrict the development of prostate cancer, but promotes metastasis in mouse tumor models [65]. As seeds for metastasis, CTC 
released by primary tumors may also account for the production and induction of several cytokines and proteins. The molecular mechanisms behind these processes are complex and many signals may interact and contribute to tumor dissemination, and ultimately, to metastasis. Among these, miRNAs are known for their capacity to work as master regulators of a large number of cellular and physiological events. Therefore, the study of miRNA deregulation in cancer can provide cues for the interpretation of events occurring during tumor spread and metastasis. An example is our recently published study in which we demonstrate that high miR-200c expression in blood from GC patients may be considered a marker associated both with poor PFS and OS [6]. Since miR200 family has been also involved in EMT [7-9], inflammatory response [11] and secretome regulation [13], we performed a pooled analysis of circulating miR- 200c levels obtained in our previous work, and GDF15 and MMP7 serum levels obtained here, in order to develop a combinatory marker for GC prognostic integrating molecular features both from CTC and microenvironment. Based on findings above depicted, we created a dichotomized variable in which low serum levels of GDF15 and MMP7, and low levels of circulating miR-200c, were grouped in a separate factor that classifies patients with good prognostic versus the remaining patients. For survival analysis, we created a second stratified variable in which high serum levels of GDF15 and MMP7, and high levels of circulating miR-200c were classified as a separate factor indicating worse survival. These variables were included in multivariate analysis in order to determine its predictive capacity on progression and death risk (Table 6). As result, the combination of high GDF15 and MMP7 serum levels with high miR-200c blood expression was established as an independent predictor of poor survival in GC patients, so this molecular signature could be related to the generation of an adverse molecular environment. In fact, the group of GC patients with high levels of 'GDF15+MMP7+miR200c' marker was enriched for metastasis $(10 / 11 ; 90.91 \% ; p=0.034$; Fisher's exact test $)$ and deaths $(10 / 11 ; 90.91 \%$; $p$ $<0.001$; log-rank test).

\section{Conclusion \& future perspective}

In this study, we found GDF15 and MMP7 significantly elevated in serum from GC patients. More importantly, these changes could be exploited to establish GDF15 and MMP7 as sensitive and specific serum biomarkers for GC diagnostic at early stages and for metastatic disease detection. In addition, high GDF15 and MMP7 serum levels were associated with poor PFS and OS. Moreover, our data suggest that concomitant elevation of GDF15 and MMP7 serum levels with high miR-200c blood expression is able to predict for short OS. Taken together, these data are sufficient to provide the basis for further studies with a larger number of patients, in order to confirm the diagnostic and prognostic capabilities of GDF15, MMP7 and miR-200c in GC. Furthermore, these findings support the importance of molecular microenvironment and its influence on the creation of favorable conditions for dissemination of disease and metastasis generation. Therefore, the combined analysis of different markers that detect several host and tumor factors may enable in future the development of more precise, sensible and specific molecular signatures for prognostic evaluation of GC patients, as well as patients with other tumors.

\section{Author contributions}

The authors were fully responsible for all content and editorial decisions, were involved at all stages of manuscript development and have approved the final version.

\section{Acknowledgements}

The authors wish to thank the patients and their relatives for their participation in this study. The essential collaboration of nurse staff and clinical data managers is also recognized. 
Financial \& competing interests disclosure

During the study, M Haz-Conde was supported in part by a grant (CA0900116) from "Instituto de Salud Carlos III" (Spain). A Figueroa was supported by "Secretaría Xeral I+D+I" (IPP0807, "Xunta de Galicia", Spain). Funding for this study was in part obtained from grants PIO61541 ("Instituto de Salud Carlos III", Spain) and PS0877 ("ServizoGalego de Saúde, Xunta de Galicia”, Spain). The authors have no other relevant affiliations or financial involvement with any organization or entity with a financial interest in or financial conflict with the subject matter or materials discussed in the present manuscript. The authors have no other relevant affiliations or financial involvement with any organization or entity with a financial interest in or financial conflict with the subject matter or materials discussed in the manuscript apart from those disclosed.

No writing assistance was utilized in the production of this manuscript.

\section{Ethical conduct of research}

The authors state that they have obtained appropriate institutional review board approval and have followed the principles outlined in the Declaration of Helsinki for all human or animal experimental investigations. In addition, for investigations involving human subjects, informed consent has been obtained from the participants involved.

\section{References}

Papers of special note have been highlighted as:

- of interest; $\bullet$ of considerable interest

1. Grivennikov SI, Greten FR, Karin M. Immunity, inflammation, and cancer. Cell 140(6), 883-899 (2010).

-• Explores in detail the connection between inflammatory and immunological cues from the tumor microenvironment and cancer and metastasis development.

2. Kim S, Takahashi H, Lin W et al. Carcinoma-produced factors activate myeloid cells through TLR2 to stimulate metastasis. Nature 457(7225), 102-106 (2009).

3. Luo J-L, Maeda S, Hsu L-C, Yagita H, Karin M. Inhibition of NF-kappaB in cancer cells converts inflammation-induced tumor growth mediated by TNFalpha to TRAIL-mediated tumor regression. Cancer Cell 6(3), 297-305 (2004).

4. Nguyen DX, Bos PD, Massague J. Metastasis: from dissemination to organ-specific colonization. Nat. Rev. Cancer 9(4), 274-284 (2009).

-• Addresses different aspects on metastasis generation, such as the molecular determinants of primary tumor that condition the dissemination of circulating tumor cells, their affinity for certain organs and tissues, and their aggressive features.

5. Madhavan D, Zucknick M, Wallwiener M et al. Circulating miRNAs as surrogate markers for circulating tumor cells and prognostic markers in metastatic breast cancer. Clin. Cancer Res. 18(1), 5972-5982 (2012).

6. Valladares-Ayerbes M, Reboredo M, Medina-Villaamil V et al. Circulating miR-200c as a diagnostic and prognostic biomarker for gastric cancer. J. Transl. Med. 10(1), 186 (2012).

-• Analyzes the diagnostic and prognostic performance of circulating miR-200c in the same cohort of gastric cancer patients analyzed for GDF15 and MMP7 serum levels in the present article.

7. Gregory PA, Bert AG, Paterson EL et al. The miR-200 family and miR-205 regulate epithelial to mesenchymal transition by targeting ZEB1 and SIP1. Nat. Cell Biol. 10(5), 593-601 (2008).

- One of first reports linking the miR-200 miRNA cluster with the regulation of the $E$ cadherin/ZEB axis and the control and maintenance of epithelial phenotype. 
8. Korpal M, Lee ES, Hu G, Kang Y. The miR-200 family inhibits epithelial-mesenchymal transition and cancer cell migration by direct targeting of E-cadherin transcriptional repressors ZEB1 and ZEB2. $J$. Biol. Chem. 283(22), 14910-14914 (2008).

9. Park S-M, Gaur AB, Lengyel E, Peter ME. The miR-200 family determines the epithelial phenotype of cancer cells by targeting the E-cadherin repressors ZEB1 and ZEB2. Genes Dev. 22(7), 894-907 (2008).

10. Gregory PA, Bracken CP, Smith E et al. An autocrine TGF-beta/ZEB/miR-200 signaling network regulates establishment and maintenance of epithelial-mesenchymal transition. Mol. Biol. Cell. 22(10), 1686-1698 (2011).

11. Rokavec M, Wu W, Luo J-L. IL6-mediated suppression of miR-200c directs constitutive activation of inflammatory signaling circuit driving transformation and tumorigenesis. Mol. Cell. 45(6), 777-789 (2012).

12. Tsui K-H, Chang Y-L, Feng T-H et al. Growth differentiation factor-15 upregulates interleukin-6 to promote tumorigenesis of prostate carcinoma PC-3 cells. J. Mol. Endocrinol. 49(2), 153-163 (2012)

13. Korpal M, Ell BJ, Buffa FM et al. Direct targeting of Sec23a by miR-200s influences cancer cell secretome and promotes metastatic colonization. Nat. Med. 17(9), 1101-1108 (2011).

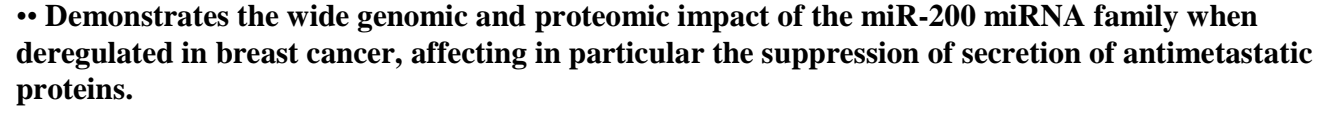

14. Fabbri M, Paone A, Calore F et al. MicroRNAs bind to Toll-like receptors to induce prometastatic inflammatory response. Proc. Natl Acad. Sci. USA 109(31), e2110-e2116 (2012).

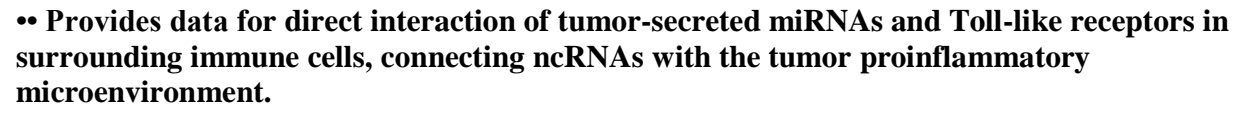

15. Crumley AB, McMillan DC, McKernan M, McDonald AC, Stuart RC. Evaluation of an inflammationbased prognostic score in patients with inoperable gastro-oesophageal cancer. Br. J. Cancer 94(5), 637641 (2006).

16. Bootcov MR, Bauskin AR, Valenzuela SM et al. MIC-1, a novel macrophage inhibitory cytokine, is a divergent member of the TGF-beta superfamily. Proc. Natl Acad. Sci. USA 94(21), 11514-11519 (1997).

17. Fairlie WD, Moore AG, Bauskin AR, Russell PK, Zhang HP, Breit SN. MIC-1 is a novel TGF-beta superfamily cytokine associated with macrophage activation. J. Leukoc. Biol. 65(1), 2-5 (1999).

18. Breit SN, Carrero JJ, Tsai VW-W et al. Macrophage inhibitory cytokine-1 (MIC-1/GDF15) and mortality in end-stage renal disease. Nephrol. Dial. Transplant. 27(1), 70-75 (2012).

19. $\mathrm{Xu} \mathrm{X,} \mathrm{Li} \mathrm{Z,} \mathrm{Gao} \mathrm{W.} \mathrm{Growth} \mathrm{differentiation} \mathrm{factor} 15$ in cardiovascular diseases: from bench to bedside Biomarkers 16(6), 466-475 (2011).

20. Wang X, Baek SJ, Eling TE. The diverse roles of nonsteroidal anti-inflammatory drug activated gene (NAG-1/GDF15) in cancer. 85, 597-606 Biochem. Pharmacol. (2012).

21. Boyle GM, Pedley J, Martyn AC et al. Macrophage inhibitory cytokine-1 is overexpressed in malignant melanoma and is associated with tumorigenicity. J. Invest. Dermatol. 129(2), 383-391 (2009).

22. Rasiah KK, Kench JG, Gardiner-Garden M et al. Aberrant neuropeptide Y and macrophage inhibitory cytokine-1 expression are early events in prostate cancer development and are associated with poor prognosis. Cancer Epidemiol. Biomarkers Prev. 15(4), 711-716 (2006).

23. Shnaper S, Desbaillets I, Brown D a et al. Elevated levels of MIC-1/GDF15 in the cerebrospinal fluid of patients are associated with glioblastoma and worse outcome. Int. J. Cancer 125(11), 2624-2630 (2009).

24. Staff AC, Bock AJ, Becker C, Kempf T, Wollert KC, Davidson B. Growth differentiation factor-15 as a prognostic biomarker in ovarian cancer. Gynecol. Oncol. 118(3), 237-243 (2010).

25. Staff AC, Trovik J, Eriksson AGZ et al. Elevated plasma growth differentiation factor-15 correlates with lymph node metastases and poor survival in endometrial cancer. Clin. Cancer Res. 17(14), 48254833 (2011)

26. Brown DA, Lindmark F, Stattin P et al. Macrophage inhibitory cytokine 1: a new prognostic marker in prostate cancer. Clin. Cancer Res. 15(21), 6658-6664 (2009).

27. Koopmann J, Buckhaults P, Brown DA et al. Serum macrophage inhibitory cytokine 1 as a marker of pancreatic and other periampullary cancers. Clin. Cancer Res. 10(7), 2386-2392 (2004).

28. Wallin U, Glimelius B, Jirstrom K et al. Growth differentiation factor 15: a prognostic marker for recurrence in colorectal cancer. Br. J. Cancer 104(10), 1619-1627 (2011). 
29. Lee DH, Yang Y, Lee SJ et al. Macrophage inhibitory cytokine-1 induces the invasiveness of gastric cancer cells by up-regulating the urokinase-type plasminogen activator system. Cancer Res. 63(15), 4648-4655 (2003).

30. Kim K-K, Lee JJ, Yang Y, You K-H, Lee J-H. Macrophage inhibitory cytokine-1 activates AKT and ERK-1/2 via the transactivation of ErbB2 in human breast and gastric cancer cells. Carcinogenesis 29(4), 704-712 (2008).

31. Park JY, Park KH, Bang S, Kim MH, Koh SS, Song SY. Expression of nonsteroidal anti-inflammatory drug-activated gene-1 (NAG-1) inversely correlates with tumor progression in gastric adenomas and carcinomas. J. Cancer Res. Clin. Oncol. 134(9), 1029-1035 (2008).

32. Baek KE, Yoon SR, Kim J-T et al. Upregulation and secretion of macrophage inhibitory cytokine-1 (MIC-1) in gastric cancers. Clin. Chim. Acta. 401(1-2), 128-133 (2009).

33. Skipworth RJE, Deans DAC, Tan BHL et al. Plasma MIC-1 correlates with systemic inflammation but is not an independent determinant of nutritional status or survival in oesophago-gastric cancer. $B r . J$. Cancer 102(4), 665-672 (2010).

34. Woessner JF, Taplin CJ. Purification and properties of a small latent matrix metalloproteinase of the rat uterus. J. Biol. Chem. 263(32), 16918-16925 (1988).

35. Page-McCaw A, Ewald AJ, Werb Z. Matrix metalloproteinases and the regulation of tissue remodelling. Nat. Rev. Mol. Cell Biol. 8(3), 221-233 (2007).

36. Gialeli $\mathrm{C}$, Theocharis AD, Karamanos NK. Roles of matrix metalloproteinases in cancer progression and their pharmacological targeting. FEBS J. 278(1), 16-27 (2011).

37. Ii M, Yamamoto H, Adachi Y, Maruyama Y, Shinomura Y. Role of matrix metalloproteinase-7 (matrilysin) in human cancer invasion, apoptosis, growth, and angiogenesis. Exp. Biol. Med. (Maywood) 231(1), 20-27 (2006).

38. Roy R, Yang J, Moses MA. Matrix metalloproteinases as novel biomarkers and potential therapeutic targets in human cancer. J. Clin. Oncol. 27(31), 5287-5297 (2009).

39. Lukaszewicz-Zając M, Mroczko B, Szmitkowski M. Gastric cancer - the role of matrix metalloproteinases in tumor progression. Clin. Chim. Acta. 412(19-20), 1725-1730 (2011).

40. Chen J-Q, Zhan W-H, He Y-L et al. Expression of heparanase gene, CD44v6, MMP-7 and nm23 protein and their relationship with the invasion and metastasis of gastric carcinomas. World $J$. Gastroenterol 10(6), 776-782 (2004).

41. Honda M, Mori M, Ueo H, Sugimachi K, Akiyoshi T. Matrix metalloproteinase-7 expression in gastric carcinoma. Gut 39(3), 444-448 (1996).

42. Yamashita K, Azumano I, Mai M, Okada Y. Expression and tissue localization of matrix metalloproteinase 7 (matrilysin) in human gastric carcinomas. Implications for vessel invasion and metastasis. Int. J. Cancer 79(2), 187-194 (1998).

43. Lee KH, Shin SJ, Kim KO et al. Relationship between E-cadherin, matrix metalloproteinase-7 gene expression and clinicopathological features in gastric carcinoma. Oncol. Rep. 16(4), 823-830 (2006).

44. Koskensalo S, Mrena J, Wiksten J-P et al. MMP-7 over expression is an independent prognostic marker in gastric cancer. Tumour Biol. 31(3), 149-155 (2010).

45. Yeh Y-C, Sheu B-S, Cheng H-C, Wang Y-L, Yang H-B, Wu J-J. Elevated serum matrix metalloproteinase-3 and -7 in $\mathrm{H}$. pylorirelated gastric cancer can be biomarkers correlating with a poor survival. Dig. Dis. Sci. 55(6), 1649-1657 (2010).

46. Mimeault M, Batra SK. Divergent molecular mechanisms underlying the pleiotropic functions of macrophage inhibitory cytokine-1 in cancer. J. Cell. Physiol. 224(3), 626-635 (2010).

47. Kapushesky M, Emam I, Holloway E et al. Gene expression atlas at the European bioinformatics institute. Nucleic Acids Res. 38(Database issue), D690-D698 (2010).

48. Lewis BP, Shih I, Jones-Rhoades MW, Bartel DP, Burge CB. Prediction of mammalian microRNA targets. Cell 115(7), 787-798 (2003).

49. Pepe MS, Etzioni R, Feng Z et al. Phases of biomarker development for early detection of cancer. $J$. Natl Cancer Inst. 93(14), 1054-1061 (2001).

50. Altman DG, McShane LM, Sauerbrei W, Taube SE. Reporting recommendations for tumor marker prognostic studies (REMARK): explanation and elaboration. BMC Med. 10, 51 (2012).

51. Camp RL, Dolled-Filhart M, Rimm DL. X-tile: a new bio-informatics tool for biomarker assessment and outcome-based cut-point optimization. Clin. Cancer Res. 10(21), 7252-7259 (2004).

52. Oken MM, Creech RH, Tormey DC et al. Toxicity and response criteria of the Eastern Cooperative Oncology Group. Am. J. Clin. Oncol. 5(6), 649-655 (1982).

53. Welsh JB, Sapinoso LM, Kern SG et al. Large-scale delineation of secreted protein biomarkers overexpressed in cancer tissue and serum. Proc. Natl Acad. Sci. USA 100(6), 3410-3415 (2003).

54. Sturgeon CM, Duffy MJ, Hofmann BR et al. National Academy of Clinical Biochemistry Laboratory Medicine Practice Guidelines for use of tumor markers in liver, bladder, cervical, and gastric cancers. Clin. Chem. 56(6), e1-e48 (2010). 
55. Szklarczyk D, Franceschini A, Kuhn M et al. The STRING database in 2011: functional interaction networks of proteins, globally integrated and scored. Nucleic Acids Res. 39(Database issue), D561D568 (2011).

56. Shi Y, Simmons MN, Seki T, Oh SP, Sugrue SP. Change in gene expression subsequent to induction of Pnn/DRS/memA: increase in p21(cip1/waf1). Oncogene 20(30), 4007-4018 (2001).

57. Johnen H, Lin S, Kuffner T et al. Tumorinduced anorexia and weight loss are mediated by the TGFbeta superfamily cytokine MIC-1. Nat. Med. 13(11), 1333-1340 (2007).

58. Ding Q, Mracek T, Gonzalez-Muniesa P et al. Identification of macrophage inhibitory cytokine-1 in adipose tissue and its secretion as an adipokine by human adipocytes. Endocrinology 150(4), 16881696 (2009).

59. Macia L, Tsai VW-W, Nguyen AD et al. Macrophage inhibitory cytokine 1 (MIC-1/GDF15) decreases food intake, body weight and improves glucose tolerance in mice on normal \& obesogenic diets. PLoS ONE 7(4), e34868 (2012).

60. Hanahan D, Weinberg RA. Hallmarks of cancer: the next generation. Cell 144(5), 646-674 (2011).

61. Lin W, Karin M. A cytokine-mediated link between innate immunity, inflammation, and cancer. $J$. Clin. Invest. 117(5), 1175-1183 (2007).

62. Wu Y, Zhou BP. Inflammation: a driving force speeds cancer metastasis. Cell Cycle 8(20), 3267-3273 (2009).

63. Chambers AF, Groom AC, MacDonald IC. Dissemination and growth of cancer cells in metastatic sites. Nat. Rev. Cancer 2(8), 563-572 (2002).

64. Kessenbrock K, Plaks V, Werb Z. Matrix metalloproteinases: regulators of the tumor microenvironment. Cell 141(1), 52-67 (2010).

-• Summarizes the role of matrix metalloproteinases in the tumor microenvironment affecting several key aspects such as cell growth, inflammatory and immune response, angiogenesis and metastasis.

65. Husaini Y, Qiu MR, Lockwood GP et al. Macrophage inhibitory cytokine-1 (MIC-1/GDF15) slows cancer development but increases metastases in TRAMP prostate cancer prone mice. PLoS ONE 7(8), e43833 (2012).

- Highlights the determinant contribution of GDF15 to the generation of metastasis in a murine model of prostate cancer.

66. Compton CC, Byrd DR, Garcia-Aguilar J, Kurtzman SH, Olawaiye A, Washington MK. AJCC Cancer Staging Atlas. Springer, NY, USA, 1-637 (2012). 